\title{
Few-cycle optical-field-induced photoemission from biased surfaces: An exact quantum theory
}

\author{
Yi Luo $\odot$, Yang Zhou $\odot$, and Peng Zhang ๑* \\ Department of Electrical and Computer Engineering, Michigan State University, East Lansing, Michigan 48824-1226, USA
}

(Received 20 July 2020; revised 19 January 2021; accepted 21 January 2021; published 4 February 2021)

\begin{abstract}
Photoemission driven by ultrafast optical fields enables spatiotemporal control of electron motion with extremely high precision. Here, we present a quantum model for ultrafast photoelectron emission from a dc-biased surface induced by laser pulses of arbitrary duration, ranging from subcycle to continuous wave, by solving the time-dependent Schrödinger equation exactly. The single formulation is valid from photon-driven electron emission in low intensity optical fields to field-driven emission in high intensity optical fields. We find the emitted charge per pulse oscillatorily increases with pulse repetition rate, due to varying coherent interaction of neighboring laser pulses. For a well-separated single pulse, our results recover the experimentally observed vanishing carrier-envelope phase sensitivity in the optical-field regime. We also find that applying a large dc field to the photoemitter is able to greatly enhance the photoemission current and in the meantime substantially shorten the current pulse.
\end{abstract}

DOI: 10.1103/PhysRevB.103.085410

\section{INTRODUCTION}

Photoelectron emission from metallic nanostructures due to ultrafast laser fields enables the spatiotemporal control of electron motion within femtosecond and nanometer scales [1-6], making it attractive to fundamental research and applications in ultrafast electron microscopy $[7,8]$, diffraction $[9,10]$, attosecond electronics $[3,11,12]$, strong-field nanooptics [13], and nanoscale vacuum devices [14-16]. The photoemission process has been extensively studied [1,3,17-36], including multiphoton emission [1,18,30,32-36], optical-field emission [6,19,37], photoassisted tunneling emission [24,25], carrier-envelope phase (CEP) sensitivity $[3,23,38,39]$, and modulation effect of two-color lasers [26-28]. Significant research efforts have been made to probe the nonlinear coherent response of metals [32-36].

A variety of models have been developed to understand the underlying emission mechanisms, such as perturbative theory [19,40,41], Floquet models [40,42], Fowler-Nordheim tunneling approximation [17,39], and directly solving the time-dependent Schrödinger equation (TDSE) $[1,3,24,40,43-$ 45]. A comprehensive study on the theoretical approaches of strong-field photoemission from surfaces is given in [40]. While there have been recent efforts to develop analytical quantum models for continuous-wave laser excitation $[24,27,28,46]$, numerical simulations are typically implemented to study photoemission due to ultrashort pulse lasers

\footnotetext{
*pz@egr.msu.edu
}

Published by the American Physical Society under the terms of the Creative Commons Attribution 4.0 International license. Further distribution of this work must maintain attribution to the author(s) and the published article's title, journal citation, and DOI.
$[3,40,44]$. Fowler-Nordheim based models are widely used to calculate the photoemission rate $[17,38,39]$, but it is only applicable in the strong optical-field regime. To explicitly reveal the interplay of various emission processes under different regimes and to systematically characterize the parametric scalings of photoemission characteristics, an exact quantum model under ultrashort pulsed condition is highly desirable.

In this paper, we construct a quantum analytical solution for ultrafast photoelectron emission from a dc-biased surface illuminated by few-cycle laser pulses (Fig. 1), by exactly solving the TDSE. Our solution is valid from the photon-driven regime to the optical-field-driven regime, and is applicable for arbitrary laser parameters (i.e., intensity, pulse duration, carrier frequency, and CEP), dc bias, and metal properties (i.e., work function and Fermi level). The model is applicable to a train of laser pulses with arbitrary pulse repetition rate. Using the analytical formulation, we examine the photoemission properties (i.e., energy spectra, photocurrent, or emission charge density) with various combinations of laser parameters and dc bias, exhibiting good agreement with the experimental observations $[3,23,39]$. This work offers clear insights into the photoelectron energy distribution and spatiotemporal dynamics of electron emission under different driving electric fields.

\section{FORMULATION}

Our one-dimensional (1D) model considers electrons with the initial energy $\varepsilon$ emitted from the metal-vacuum interface at $x=0$, under a dc electric field $F_{0}$, and an optical electric field (Fig. 1) of a Gaussian laser pulse train with a time period $T=2 L$ of the form,

$$
\begin{aligned}
F(t) & =F_{1} e^{-t^{2} / \sigma^{2}} \cos (\omega t+\phi), \\
(2 l-1) L & <t \leqslant(2 l+1) L \quad \text { with } \quad l=0, \pm 1, \pm 2, \ldots,
\end{aligned}
$$


(a)

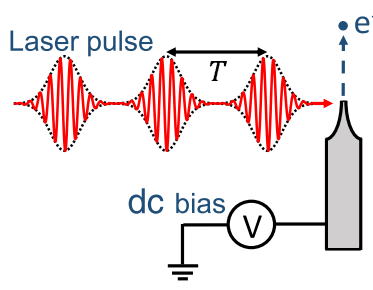

(b) Field strength

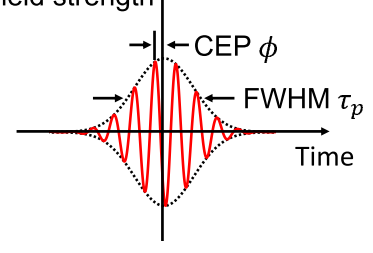

FIG. 1. (a) Sketch of photoelectron emission from a biased emitter under the illumination of a laser pulse train with a time period $T$. (b) A single laser pulse with carrier-envelope phase (CEP) $\phi$ and full width at half maximum (FWHM) of the field envelope $\tau_{p}$. The red curve and black dotted lines denote the time evolution of the laser electric field and laser pulse envelope, respectively.

where $F_{1}$ is the peak of optical-field strength, $\sigma=$ $\tau_{p} /(2 \sqrt{\ln 2}) \cong \tau_{p} / 1.665$ with $\tau_{p}$ being the full width at half maximum (FWHM) of the field envelope, $\omega$ is the angular frequency of the carrier wave, and $\phi$ is the CEP. All the laser pulses are CEP stabilized with $\omega=m \pi / L=m \omega_{E}$, with $m$ being a positive integer and $\omega_{E}$ the pulse repetition frequency [47]. When $L / \tau_{p} \gg 1$, the temporal interaction between consecutive laser pulses becomes negligible and $F(t)$ can be used to study photoemission due to a single laser pulse. By taking the Fourier series, the laser field in Eq. (1) can be expressed as,

$$
F(t)=F_{1} a_{0} \cos \phi+\sum_{\substack{n=-\infty \\ n \neq 0}}^{+\infty} F_{1} a_{n} \cos \left(n \omega_{E} t+\phi\right)
$$

where $\quad a_{0}=\frac{1}{L} \int_{0}^{L} e^{-t^{2} / \sigma^{2}} \cos \left(m \omega_{E} t\right) d t, \quad$ and $\quad a_{n}=$ $\frac{1}{L} \int_{0}^{L} e^{-t^{2} / \sigma^{2}} \cos \left[(n-m) \omega_{E} t\right] d t$. From Eq. (2), it is clear that the incident laser pulse train is a superposition of sine waves with frequencies separated by $\omega_{E}$. We assume the laser electric field is spatially uniform and perpendicular to the metal surface; thus the time-dependent potential barrier near the meatal-vacuum interface is $[24,27,28,46,48]$,

$$
\Phi(x, t)= \begin{cases}0, & x<0 \\ E_{F}+W_{\mathrm{eff}}-e F_{0} x-e F(t) x, & x \geqslant 0\end{cases}
$$

where $E_{F}$ is the Fermi energy of the metal cathode; $W_{\text {eff }}=$ $W-2 \sqrt{e^{3} F_{0} / 16 \pi \varepsilon_{0}}$ is the effective work function with Schottky effect [24,28], with $W$ being the nominal work function; $e$ is the elementary charge; $\varepsilon_{0}$ is the free space permittivity; and $F(t)$ is given by Eq. (2).

To get the electron emission probability, we solve the TDSE,

$$
i \hbar \frac{\partial \psi(x, t)}{\partial t}=-\frac{\hbar^{2}}{2 m_{e}} \frac{\partial^{2} \psi(x, t)}{\partial x^{2}}+\Phi(x, t) \psi(x, t)
$$

where $\hbar$ is the reduced Plank constant, $\psi(x, t)$ is the electron wave function, $m_{e}$ is the electron mass, and $\Phi(x, t)$ is the potential energy given in Eq. (3). For $x \geqslant 0$ (in the vacuum), the exact solution of the electron wave function is found to be (see the Appendixes for details),

$$
\begin{aligned}
\psi(x, t)= & \sum_{l=-\infty}^{\infty} T_{l}\left[\operatorname{Ai}\left(-\eta_{l}\right)-i \operatorname{Bi}\left(-\eta_{l}\right)\right] \times \exp \left(-i \frac{\varepsilon}{\hbar} t-i l \omega_{E} t\right) \\
& \times \exp \left(\frac{i e F_{1}}{\hbar} G x+\frac{i e^{2} F_{1}^{2}}{8 \hbar m_{e}} M-\frac{i e^{2}\left(F_{0}+F_{1} a_{0} \cos \phi\right) F_{1}}{\hbar m_{e}} N-\frac{i e^{2} F_{1}^{2}}{4 \hbar m_{e} \omega_{E}^{2}} R\right), \quad x \geqslant 0,
\end{aligned}
$$

where $\varepsilon$ is the electron initial energy, $\quad G=\sum_{n=-\infty, n \neq 0}^{+\infty} \frac{a_{n} \sin \left(n \omega_{E} t+\phi\right)}{n \omega_{E}} ; \quad M=\sum_{n=-\infty, n \neq 0}^{+\infty} \frac{a_{n}^{2} \sin \left(2 n \omega_{E} t+2 \phi\right)+a_{n} a_{-n} \sin \left(2 n \omega_{E} t\right)}{n^{3} \omega_{E}^{3}}$; $N=\sum_{n=-\infty, n \neq 0}^{+\infty} \frac{a_{n} \sin \left(n \omega_{E} t+\phi\right)}{n^{3} \omega_{E}^{3}} ; R=\sum_{n=-\infty, n \neq 0}^{+\infty} \sum_{m=-\infty, m \neq 0, n,-n}^{+\infty} \frac{a_{m} a_{n}}{m n}\left\{\frac{\sin \left[(n-m) \omega_{E} t\right]}{(n-m) \omega_{E}}-\frac{\sin \left[(n+m) \omega_{E} t+2 \phi\right]}{(n+m) \omega_{E}}\right\} ; \eta_{l}=\left[\frac{n^{3} \omega_{E}}{e\left(F_{0}+F_{1} a_{0} \cos \phi\right)}+x+\right.$ $\left.\sum_{n=-\infty, n \neq 0}^{+\infty} \frac{e F_{1} a_{n} \cos \left(n \omega_{E} t+\phi\right)}{n^{2} \omega_{E}^{2} m_{e}}\right]\left[\frac{2 e m_{e}\left(F_{0}+F_{1} a_{0} \cos \phi\right)}{\hbar^{2}}\right]^{1 / 3}$; the drift kinetic energy $E_{l}=\varepsilon+l \hbar \omega_{E}-E_{F}-W_{\text {eff }}-U_{p}$; the ponderomotive energy $U_{p}=\sum_{n=-\infty, n \neq 0}^{+\infty} \frac{e^{2} F_{1}^{2}\left[a_{n}^{2}+a_{n} a_{n} \cos (2 \phi)\right]}{4 m_{e} n^{2} \omega_{E}^{2}} ; \mathrm{Ai}$ and $\mathrm{Bi}$ are the Airy functions of the first kind and second kind, respectively, showing an outgoing wave traveling towards the $+x$ direction [24,49,50]; and $T_{l}$ represents the transmission coefficient.

For $x<0$ (inside the cathode), the exact solution of Eq. (4) is,

$$
\psi(x, t)=\exp \left(-\frac{i \varepsilon t}{\hbar}+i k_{0} x\right)+\sum_{l=-\infty}^{\infty} R_{l} \exp \left(-i \frac{\varepsilon+l \hbar \omega_{E}}{\hbar} t-i k_{l} x\right), \quad x<0
$$

which shows the superposition of an incident plane wave with initial energy $\varepsilon$ and a set of reflected plane waves with reflection coefficient $R_{l}$ and energies $\varepsilon+l \hbar \omega_{E}$, where the wave numbers $k_{0}=\sqrt{2 m_{e} \varepsilon / \hbar^{2}}$ and $k_{l}=\sqrt{2 m_{e}\left(\varepsilon+l \hbar \omega_{E}\right) / \hbar^{2}}$.

Following the probability current density $J(x, t)=$ $\left(i \hbar / 2 m_{e}\right)\left(\psi \partial \psi^{*} / \partial x-\psi^{*} \partial \psi / \partial x\right)=\left(i \hbar / 2 m_{e}\right) \sum_{n=-\infty}^{\infty}$ $\sum_{l=-\infty}^{\infty}\left(\psi_{n} \partial \psi_{l}^{*} / \partial x-\psi_{n}^{*} \partial \psi_{l} / \partial x\right)$, the normalized emission current density is defined as the ratio of the transmitted probability current density over the incident probability current density, $\quad w(\varepsilon, x, t)=J_{t}(\varepsilon, x, t) / J_{i}(\varepsilon, x, t)$. Thus, we obtain, in nondimensional quantities [24,27,28], $\bar{\varepsilon}=\varepsilon / W_{\mathrm{eff}}, \quad \bar{\omega}_{E}=\omega_{E} \hbar / W_{\mathrm{eff}}, \quad \bar{t}=t W_{\mathrm{eff}} / \hbar, \quad \bar{E}_{F}=E_{F} / W_{\mathrm{eff}}$, $\bar{x}=x / \lambda_{0}, \quad \lambda_{0}=\sqrt{\hbar^{2} / 2 m_{e} W_{\text {eff }}}, \quad \bar{F}_{0}=F_{0} e \lambda_{0} / W_{\text {eff }}, \quad \bar{F}_{1}=$ $F_{1} e \lambda_{0} / W_{\text {eff }}, \bar{U}_{p}=U_{p} / W_{\text {eff }}$, and the normalized instantaneous 
photoemission current density is

$$
w(\bar{\varepsilon}, \bar{x}, \bar{t})=\frac{1}{\sqrt{\bar{\varepsilon}}} \sum_{n=-\infty}^{\infty} \sum_{l=-\infty}^{\infty} \operatorname{Im}\left[e^{i(l-n) \bar{\omega}_{E} \bar{t}} T_{n} T_{l}^{*}(\bar{C}+i \bar{D})\right],
$$

where $\quad \bar{C}=\bar{F}_{1}\left[\operatorname{Ai}\left(-\bar{\eta}_{l}\right) \operatorname{Bi}\left(-\bar{\eta}_{n}\right)-\operatorname{Ai}\left(-\bar{\eta}_{n}\right) \operatorname{Bi}\left(-\bar{\eta}_{l}\right)\right] \bar{G}+$ $\left(\bar{F}_{0}+\bar{F}_{1} a_{0} \cos \phi\right)^{1 / 3}\left[\mathrm{Ai}\left(-\bar{\eta}_{n}\right) \mathrm{Ai}^{\prime}\left(-\bar{\eta}_{l}\right)+\mathrm{Bi}\left(-\bar{\eta}_{n}\right) \mathrm{Bi}^{\prime}\left(-\bar{\eta}_{l}\right)\right]$, $\bar{D}=\bar{F}_{1}\left[\mathrm{Ai}\left(-\bar{\eta}_{n}\right) \mathrm{Ai}\left(-\bar{\eta}_{l}\right)+\mathrm{Bi}\left(-\bar{\eta}_{n}\right) \mathrm{Bi}\left(-\bar{\eta}_{l}\right)\right] \bar{G}+\left(\bar{F}_{0}+\right.$ $\left.\bar{F}_{1} a_{0} \cos \phi\right)^{1 / 3}\left[\mathrm{Ai}\left(-\bar{\eta}_{n}\right) \mathrm{Bi}^{\prime}\left(-\bar{\eta}_{l}\right)-\operatorname{Bi}\left(-\bar{\eta}_{n}\right) \mathrm{Ai}^{\prime}\left(-\bar{\eta}_{l}\right)\right], \quad \bar{G}=$ $\sum_{n=-\infty, n \neq 0}^{+\infty} \frac{a_{n} \sin \left(n \bar{\omega}_{E} \bar{t}+\phi\right)}{n \bar{\omega}_{E}}, \quad$ and $\quad \bar{\eta}_{n}=\left(\bar{F}_{0}+\bar{F}_{1} a_{0} \cos \phi\right)^{1 / 3} \times$ $\left[\frac{\bar{\varepsilon}+n \bar{\omega}_{E}-\bar{E}_{F}-\bar{U}_{p}-1}{\bar{F}_{0}+\bar{F}_{1} a_{0} \cos \phi}+\sum_{l=-\infty, l \neq 0}^{+\infty} \frac{2 \bar{F}_{1} a_{l} \cos \left(l \bar{\omega}_{E} \bar{t}+\phi\right)}{l^{2} \bar{\omega}_{E}^{2}}+\bar{x}\right]$. The normalized time-averaged photoemission current density over one laser pulse is defined as the ratio of the total emission charge density $Q$ due to a single laser pulse and the length $\tau_{p}$,

$$
\begin{aligned}
\langle w(\bar{\varepsilon})\rangle & =\frac{Q}{\tau_{p}}=\sum_{n=-\infty}^{\infty}\left\langle w_{n}(\bar{\varepsilon})\right\rangle, \\
\left\langle w_{n}(\bar{\varepsilon})\right\rangle & =\left|T_{n}\right|^{2} \frac{\left(\bar{F}_{0}+\bar{F}_{1} a_{0} \cos \phi\right)^{1 / 3}}{\pi \sqrt{\bar{\varepsilon}}} \frac{2 L}{\tau_{p}},
\end{aligned}
$$

where $\left\langle w_{n}(\bar{\varepsilon})\right\rangle$ denotes the time-averaged emission current density through the $n$th channel, with emitted electron energy $\varepsilon+n \hbar \omega_{E}$. The transmission coefficient $T_{n}$ (and reflection coefficient $R_{n}$ ) can be obtained from boundary conditions that both the electron wave function $\psi(x, t)$ and its derivative $\partial \psi(x, t) / \partial x$ are continuous at $x=0$ (see the Appendixes for details).

For the special case of dc field $F_{0}=0$ and CEP $\phi=\pi / 2$, the electron wave function for $x \geqslant 0$ is revised by displacing $\left[\mathrm{Ai}\left(-\eta_{l}\right)-i \operatorname{Bi}\left(-\eta_{l}\right)\right]$ in Eq. (5) with $\exp \left\{i\left[x+\sum_{n=-\infty, n \neq 0}^{+\infty} \frac{e F_{1} a_{n} \cos \left(n \omega_{E} t+\phi\right)}{n^{2} \omega_{E}^{2} m_{e}}\right] \sqrt{2 m_{e} E_{l}} / \hbar\right\}$. The normalized photoemission current density becomes,

$$
\begin{aligned}
w(\bar{\varepsilon}, \bar{x}, \bar{t}) & =\frac{1}{\sqrt{\bar{\varepsilon}}} \sum_{n=-\infty}^{\infty} \sum_{l=-\infty}^{\infty} \operatorname{Im}\left[i e^{i(l-n) \bar{\omega}_{E} \bar{t}} T_{n} T_{l}^{*} \bar{A} e^{i \bar{B}}\right], \\
\langle w(\bar{\varepsilon})\rangle & =\sum_{n=-\infty}^{\infty}\left\langle w_{n}(\bar{\varepsilon})\right\rangle, \quad\left\langle w_{n}(\bar{\varepsilon})\right\rangle=\frac{\operatorname{Re}\left(\left|T_{n}\right|^{2} \sqrt{\bar{E}_{n}}\right)}{\sqrt{\bar{\varepsilon}}} \frac{2 L}{\tau_{p}},
\end{aligned}
$$

with $\bar{A}=\left(\sqrt{\bar{E}_{l}}\right)^{*}+\sum_{m=-\infty, m \neq 0}^{+\infty} \frac{\bar{F}_{1} a_{m} \sin \left(m \bar{\omega}_{E} \bar{t}+\phi\right)}{m \bar{\omega}_{E}}$ and $\bar{B}=$ $\left[\sqrt{\bar{E}_{n}}-\left(\sqrt{\bar{E}_{l}}\right)^{*}\right]\left(\bar{x}+\sum_{m=-\infty, m \neq 0}^{+\infty} \frac{2 \bar{F}_{1} a_{m} \cos \left(m \bar{\omega}_{E} \bar{t}+\phi\right)}{m^{2} \bar{\omega}_{E}^{2}}\right.$ (see the Appendixes for details for the calculation of $T_{n}$ ).

\section{RESULTS}

Based on the analytical formulation presented above, we provide an analysis of the photoelectron emission with different laser and dc fields. Unless specified otherwise, the default value of the laser wavelength is $800 \mathrm{~nm}$ (or laser period $T_{\omega}$ of $2.67 \mathrm{fs}$ ); the cathode metal is assumed to be gold $[19,24,40]$, with Fermi energy $E_{F}=5.53 \mathrm{eV}$ and work function $W=$ $5.1 \mathrm{eV}$; the pulse width $\tau_{p}=8.8$ fs (i.e., $\sim 3$ cycles); the laser pulse repetition period $T=267 \mathrm{fs}\left(\gg \tau_{p}\right.$ to isolate a single laser pulse and avoid temporal interaction between adjacent laser pulses); the CEP $\phi=0$; the dc field $F_{0}=1 \times 10^{4} \mathrm{~V} / \mathrm{m}$; and the peak laser field $F_{1}=1 \times 10^{9} \mathrm{~V} / \mathrm{m}$. Since most of the (a)

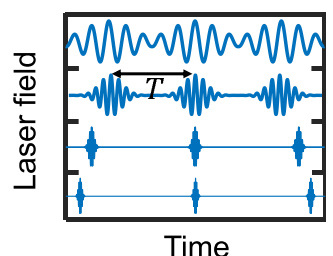

(c)

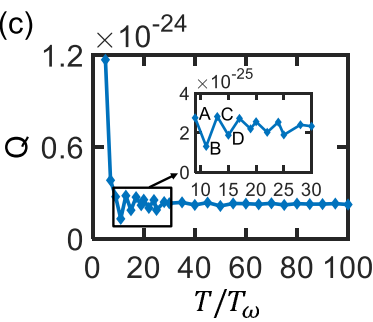

(b)

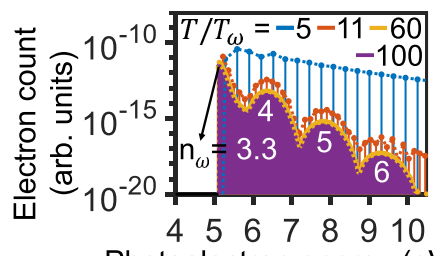

(d)

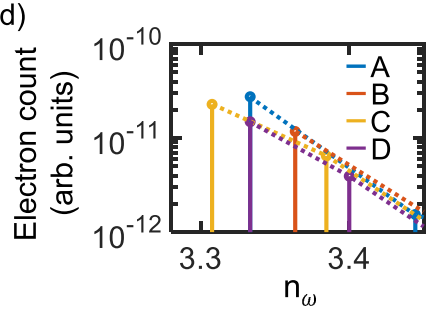

FIG. 2. Effects of time separation $T$ between adjacent laser pulses on photoelectron energy spectra and total emission charge density $Q$. (a) Laser electric field for different $T$. From top to bottom, $T=13,29,160$, and $267 \mathrm{fs}$, corresponding to $T / T_{\omega}=5,11,60$, and 100, respectively. (b) Energy spectra for different $T . n_{\omega}$ denotes the laser photon order (with single photon energy $\hbar \omega=1.55 \mathrm{eV}$ ). (c) $Q$ as a function of $T / T_{\omega}$. The inset shows the magnification of (c) between $T / T_{\omega}=9$ and 30, where A-D denote $T / T_{\omega}=9,11,13$, and 15, respectively. (d) Photoelectron energy spectra near the maximum at A-D in the inset of (c).

electrons are emitted with initial energies near the Fermi level [24,40,50,51], we choose the electron initial energy $\varepsilon=E_{F}$ for simplicity.

In Fig. 2, we show the photoelectron energy spectra and total emission charge density $Q\left(=\langle w\rangle \tau_{p}\right)$ for various time intervals $T$ between adjacent laser pulses. It is found that as $T$ decreases, photoelectron emission is gradually confined to a smaller number of emission channels but with more electron yield, because of the decreasing frequency ratio between laser carrier $\omega$ and pulse repetition frequency $\omega_{E}$ [Fig. 2(b)]. The interaction of consecutive laser pulses leads to the smearing of multiphoton absorption peaks (with respect to laser photon energy $\hbar \omega$ ) in the photoelectron energy spectra envelope with decreasing $T$. Figure 2(c) shows that when $T / T_{\omega}<9$ the closely spaced laser pulse train induces a total emission charge $Q$ per pulse that is significantly higher than that due to a well-separated single laser pulse. When $T$ increases, $Q$ decreases and eventually becomes independent of $T$ when $T / T_{\omega}>60$, indicating the laser pulses are well separated and the results may be regarded as being from a single laser pulse. It is interesting to note the oscillatory feature of $Q$ in the range of $9<T / T_{\omega}<60$ [enlarged in the inset of Fig. 2(c)], due to the varying coherent interaction between neighboring pulses. Figure 2(d) compares the photoelectron energy spectra with different $T$ in this oscillation regime [cf. A-D in the inset of Fig. 2(c)], where both the peak electron emission yield and the dominant emission channel vary with $T$.

We evaluate the effect of CEP $\phi$ on the photoelectron energy spectra and total emission current density $\langle w\rangle$ for different pulse durations $\tau_{p}$ in Fig. 3. For small $\tau_{p}$ (e.g., 4.4 fs, or 1.7 optical cycles), the spectral features are sinusoidally modulated with $\phi$ [Fig. 3(b)]. This is consistent with the 
(a)

$$
\phi=-0-\pi
$$
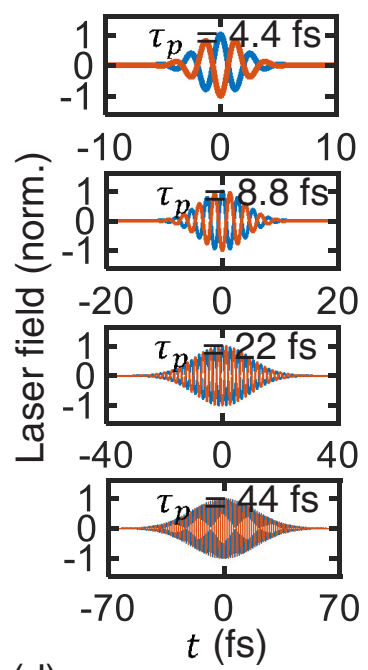

(d)

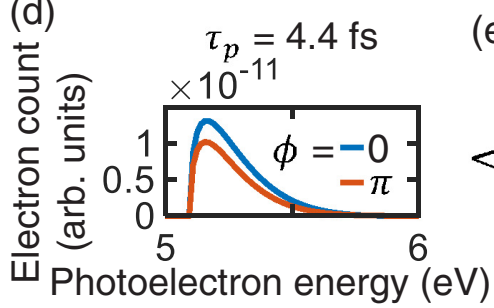

(b) $10^{-17} 10^{-11}$ Electron count (arb. units)
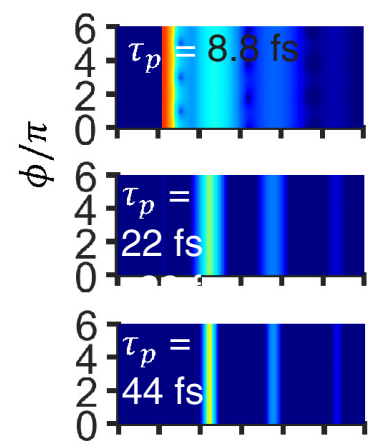

45678910 Photoelectron energy $(\mathrm{eV})$

(e)

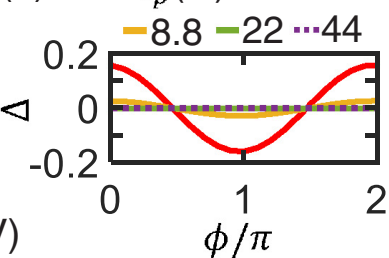

(c)

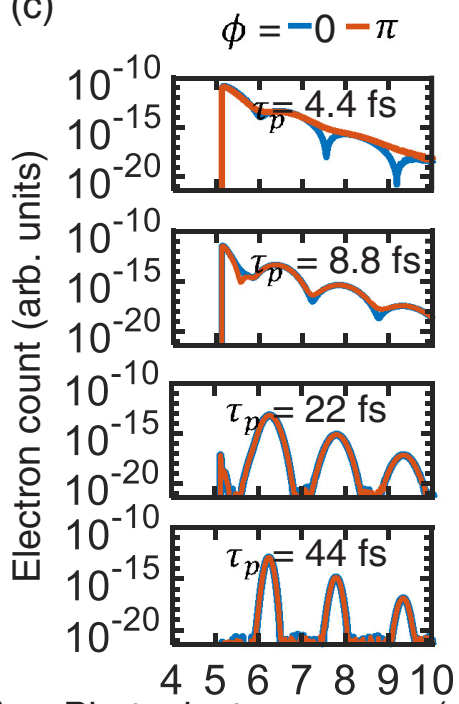

Photoelectron energy (eV)

(f)

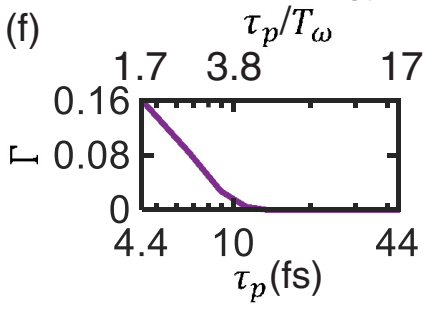

FIG. 3. CEP modulation in energy spectra with different pulse duration $\tau_{p}$. (a) Laser electric field for different $\tau_{p}$ when CEP $\phi=0$ and $\pi$. (b) Energy spectra as a function of $\phi$ for different $\tau_{p}$. (c) Extracted energy spectra of $\phi=0$ and $\pi$ from (b). (d) Linear plot of energy spectrum for $\tau_{p}=4.4 \mathrm{fs}$ in (c). (e) Normalized current modulation magnitude $\Delta=\left(\langle w\rangle-\langle w\rangle_{\text {ave }}\right) /\langle w\rangle_{\text {ave }}$ as a function of $\phi$ for different $\tau_{p}$. Here, $\langle w\rangle_{\text {ave }}=\left(\langle w\rangle_{\max }+\langle w\rangle_{\min }\right) / 2$ denotes the averaged value of $\langle w\rangle$ with respect to $\phi$. (f) Current modulation depth $\Gamma=\left(\langle w\rangle_{\max }-\langle w\rangle_{\min }\right) /\left(\langle w\rangle_{\max }+\langle w\rangle_{\min }\right)$ as a function of $\tau_{p}$.

experimental observation of CEP modulation in photoemission spectra with a few-cycle laser pulse [3,23]. As pulse width $\tau_{p}$ increases, the CEP modulation on spectra becomes less pronounced, and the multiphoton peaks in the spectra become narrower [Fig. 3(c)], gradually approaching those from continuous-wave excitation [24]. The energy spectra for small $\tau_{p}(=4.4 \mathrm{fs})$ are enlarged in Fig. 3(d), showing a higher electron yield and broader spectrum for $\phi=0$ than for $\phi=\pi$. Figures 3(e) and 3(f) show that CEP modulation is important for a short laser pulse up to $\tau_{p} / T_{\omega} \sim 4$ and the modulation of total photoemission current with $\phi$ decreases for longer pulse width $\tau_{p}$.

We next examine the CEP sensitivity of photoelectron emission charge $Q$ under different laser fields $F_{1}$. As shown in Figs. 4(a) and 4(b), in general, the CEP modulation on $Q$ increases as $F_{1}$ increases. Also, the position of $\phi$ for the maximum (or minimum) $Q$ shifts when the laser field $F_{1}$ increases for a fixed pulse duration $\tau_{p}$ [see the dashed lines in Figs. 4(a) and 4(b)]. To investigate the CEP sensitivity more closely, in Fig. 4(c), we plot the difference between the maximum and minimum values of $Q$ in the curves of Figs. 4(a) and 4(b) as a function of $F_{1}$. Following [39], by fitting the $Q$ vs $\phi$ curves with a sinusoidal function of $\cos (\phi+\angle Q)$, we can identify the phase shift of the maximum $Q$ with $F_{1}$, as shown in Fig. 4(d). We see a pronounced dip in $Q_{\max }-Q_{\min }$ at large laser field $F_{1}=9 \mathrm{~V} / \mathrm{nm}$ for both cases of $\tau_{p}=4.7$ and $8.8 \mathrm{fs}$, and for $\tau_{p}=8.8 \mathrm{fs}$ another dip appearing at $F_{1}=5 \mathrm{~V} / \mathrm{nm}$ [cf. Fig. 4(c)]. From Fig. 4(d), phase shifts of $\pi$ in $\phi$ are found near these dips. These behaviors agree very well with the vanishing
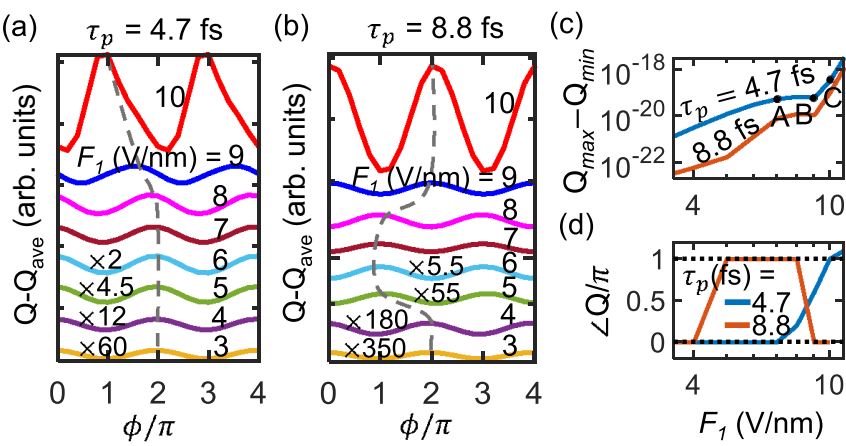

FIG. 4. CEP sensitivity of total emission charge density $Q$ under different laser fields $F_{1}$. (a), (b) Difference between $Q$ and its averaged value $Q_{\text {ave }}$ as a function of $\phi$ for different $F_{1}$ with pulse duration (a) $\tau_{p}=4.7$ fs and (b) $\tau_{p}=8.8$ fs. For a given $F_{1}$, $Q_{\text {ave }}=\left(Q_{\max }+Q_{\min }\right) / 2$. Dashed lines indicate the shift of the phase for the CEP modulation. (c) Difference between the maximum and minimum values of charge $Q_{\max }-Q_{\min }$ in the curves of (a) and (b), as a function of $F_{1}$ for different $\tau_{p}$. Points A-C denote $F_{1}=7,9$, and 10 $\mathrm{V} / \mathrm{nm}$, respectively. (d) Photoemission charge phase $\angle Q$ as a function of $F_{1}$ for different $\tau_{p} . \angle Q$ is obtained by using $B \cos (\phi+\angle Q)$ to fit the curves in (a), (b), with $B=Q_{\max }-Q_{\min }$. 

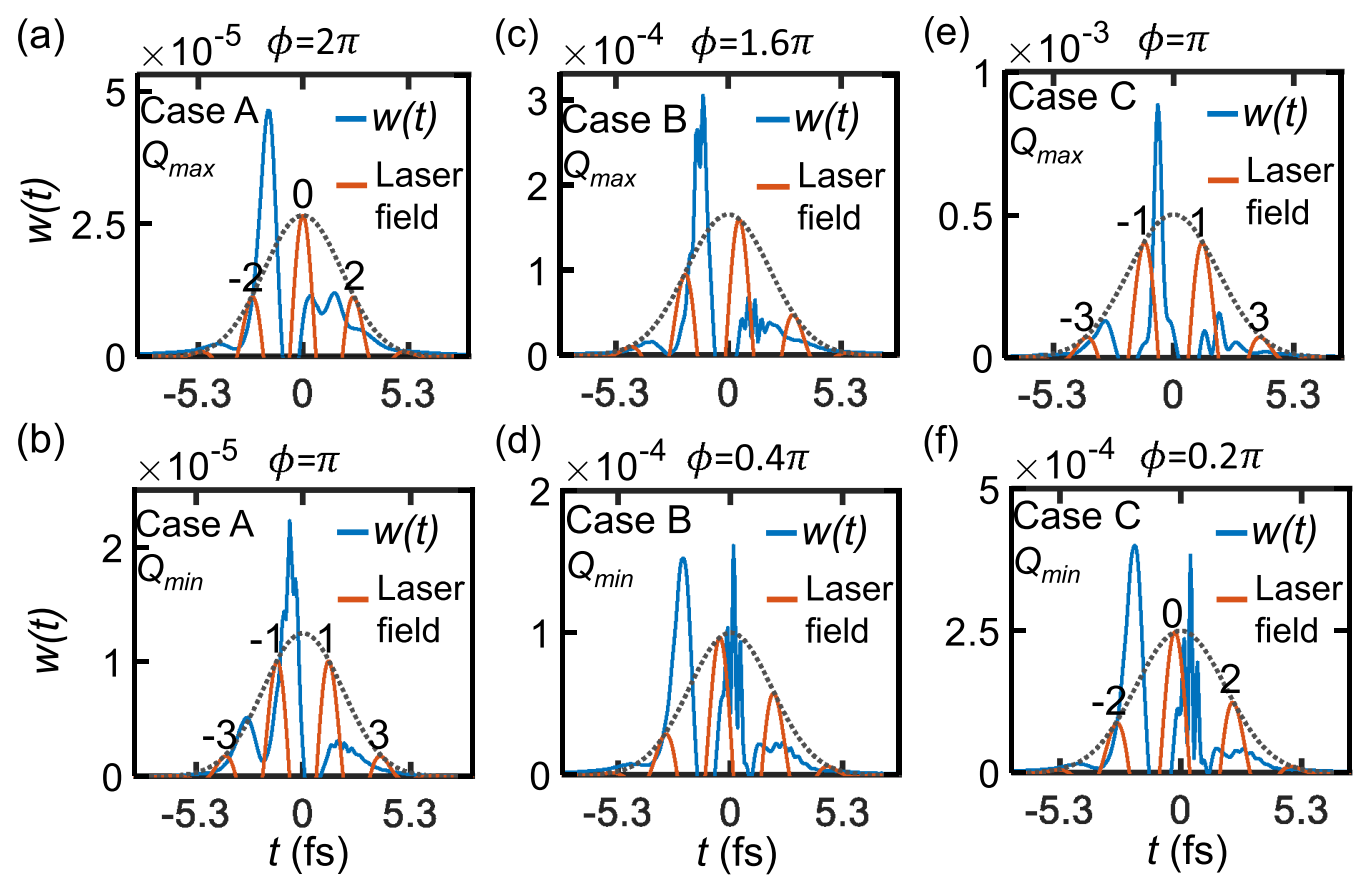

FIG. 5. Time-dependent emission current density $w(t)$ at the surface $(\bar{x}=0$, with surface oscillatory current excluded) as a function of time $t$ for pulse duration $\tau_{p}=4.7 \mathrm{fs}$ at CEP $\phi$ when $Q_{\max }$ (top row) or $Q_{\min }$ (bottom row) occurs, under different laser fields at (a), (b) $F_{1}=7$ $\mathrm{V} / \mathrm{nm}$; (c), (d) $F_{1}=9 \mathrm{~V} / \mathrm{nm}$; and (e), (f) $F_{1}=10 \mathrm{~V} / \mathrm{nm}$. The values of laser field $F_{1}$ correspond to cases A-C in Fig. 4(c), respectively. The value of CEP $\phi$ in each panel corresponds to the occurrence of $Q_{\max }$ (top row) or $Q_{\min }$ (top row) in Fig. 4(a). The blue lines are for emission current density, red lines for laser field, and black dotted lines for laser pulse envelope. The optical half cycles of the laser field in (a), (b), (e), (f) are numbered as $0, \pm 1, \pm 2$, and \pm 3 , with " 0 " being the center cycle with the highest peak. Only positive optical half cycles are shown.

CEP sensitivity of photoemission accompanied by a $\pi$ phase shift in the optical-field regime observed in recent experiments [39].

To uncover the physical origin of the vanishing CEP sensitivity behavior and the CEP phase shift in the photoemission charge, we plot the time-dependent electron emission current density $w(t)$ at the surface $(\bar{x}=0)$ as a function of time, under different laser fields $F_{1}$ and CEP $\phi$ for $\tau_{p}=4.7 \mathrm{fs}$, as shown in Fig. 5. The laser field strengths of 7, 9, and $10 \mathrm{~V} / \mathrm{nm}$ used in Fig. 5 correspond to cases A-C in Fig. 4(c), respectively. By observing these time-dependent current pulses, it is clear that electron emission starts at the beginning of each positive half cycle in a given laser field pulse. When $F_{1}=7 \mathrm{~V} / \mathrm{nm}$ [case A before the dip in CEP sensitivity in Fig. 4(c)], evennumbered positive optical half cycles [Fig. 5(a)] drive more photoelectron emission than odd-numbered positive optical half cycles [Fig. 5(b)]. However, as the laser field $F_{1}$ is increased to $10 \mathrm{~V} / \mathrm{nm}$ [case $\mathrm{C}$ after the dip in CEP sensitivity in Fig. 4(c)], odd-numbered positive half cycles trigger more electron emission than even-numbered cycles [cf. Figs. 5(e) and 5(f)]. This indicates that in the strong field regime there exists a competition between even and odd positive halfcycle contributions to photoelectron emission, and thus a $\pi$ phase shift in $\phi$ as shown in Fig. 4(d), with varying CEP. At $F_{1}=9 \mathrm{~V} / \mathrm{nm}$ [case $\mathrm{B}$ at the dip in CEP sensitivity in Fig. 4(c) ], $Q_{\max }-Q_{\min }$ becomes minimal, where $Q_{\max }$ and $Q_{\min }$ occur at $\phi=1.6 \pi$ and $0.4 \pi$, respectively. The competition between electron emission from different positive optical half cycles also leads to the dips in CEP sensitivity and phase shifts at $F_{1}=5$ and $9 \mathrm{~V} / \mathrm{nm}$ for $\tau_{p}=8.8$ fs in Figs. 4(c) and $4(\mathrm{~d})$.

It is important to note that, for clarity, we plot in Fig. 5 only the emitted current density that eventually escapes from the surface, whereas the local strong oscillatory current density near the surface typically associated with photoemission (e.g., see Figs. 6(a) and 6(b) below, and also Refs. [22,24,27,40,52]) is filtered out. This is possible in our exact analytical calculation using Eq. (9) by excluding the high $n$-order (and $l$-order) terms, which is verified to give the strong oscillatory surface currents only. This is also consistent with previous study showing the high energy regime in the photoelectron spectra is due to surface oscillations and rescattering (cf. Fig. 4 in Ref. [22]).

It is also noteworthy that, though electron emission starts at the beginning of every positive optical half cycle in the laser pulse, there is typically a time delay between the peak of the positive optical half cycle and the peak of the emission current pulse, as seen in Fig. 5. Furthermore, a stronger positive optical half cycle does not necessarily lead to a higher current pulse emission, which, however, depends strongly on the emission from neighboring half cycles in a laser pulse. These observations indicate that further examination is needed on the validity of the widely used Fowler-Nordheim rate equations, in which current emission follows closely the optical positive half cycles, to study the CEP sensitive, time-dependent strongfield photoemission [39]. 

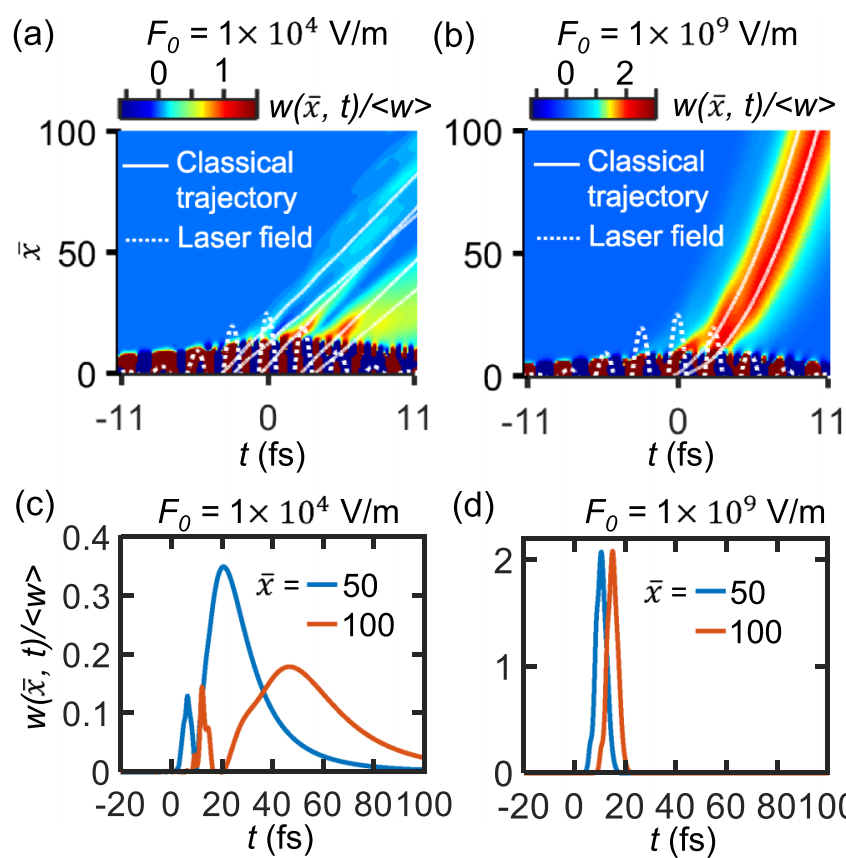

(d)

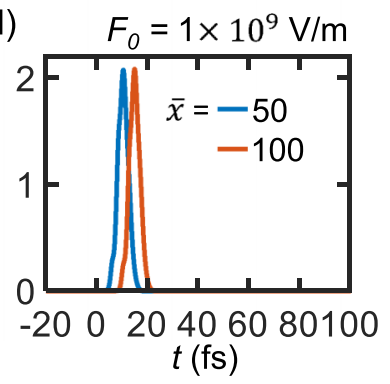

FIG. 6. Total time-dependent emission current density $w(\bar{x}, t)$ under the dc field $F_{0}=1 \times 10^{4}$ and $1 \times 10^{9} \mathrm{~V} / \mathrm{m}$. (a), (b) $w(\bar{x}, t)$ including surface oscillation currents as a function of the space $\bar{x}$ and time $t$. Solid white lines show the corresponding classical trajectories. Dotted white lines show the positive half cycles of the laser electric field. (c), (d) Emission current density $w(t)$ at $\bar{x}=50$ and 100 , as a function of time $t$. The time-dependent current in all figures is normalized in terms of the time-averaged emission current density $\langle w\rangle$. Here, the laser pulse duration $\tau_{p}=8.8 \mathrm{fs}$ and the peak laser field $F_{1}=1 \mathrm{~V} / \mathrm{nm}$. When $F_{0}=1 \times 10^{4} \mathrm{~V} / \mathrm{m},\langle w\rangle=2.5 \times 10^{-11}$; when $F_{0}=1 \times 10^{9} \mathrm{~V} / \mathrm{m},\langle w\rangle=2.1 \times 10^{-7}$.

In Fig. 6, we plot the total time-dependent photoemission current density $w(\bar{x}, t)$, including oscillatory surface currents, as a function of the space $\bar{x}$ and time $t$ under different $\mathrm{dc}$ bias. The strong oscillatory surface currents are confined to the very limited region near the surface only. It is found that increasing the dc field from $F_{0}=1 \times 10^{4}$ to $1 \times 10^{9}$ $\mathrm{V} / \mathrm{m}$ increases the time-averaged emission current density from $\langle w\rangle=2.5 \times 10^{-11}$ to $2.1 \times 10^{-7}$. More importantly, the emission current pulse is significantly shortened [from 19.7 to $4.8 \mathrm{fs}$ of full width at half maximum (FWHM) at $\bar{x}=50$ ]. Also, due to the strong acceleration of the larger $F_{0}$, the shape of the pulse is retained (without consideration of space charge effect) as the current pulse travels further from the surface [Fig. 6(d)]. This may provide a practical way to shorten the photoemission current pulse by simply adding a large dc bias. The solid white lines are the corresponding classical trajectories [40], showing good agreement with the electron dynamics from our quantum model. Note because of the relatively small optical field used, the trajectories of the emitted photoelectrons in Fig. 6(a) show fewer oscillatory features compared to those cases with strong laser fields (cf. Fig. 3 of Ref. [52]). This is due to the weaker backpropagation and acceleration processes of emitted electrons (i.e., smaller quiver motion) under weak laser electric fields. When adding a strong dc bias, most of the electrons are able to escape from the metal surface with negligible quiver motion, as shown in Fig. 6(b), similar to the dc field emission process. The classical trajectories in Figs. 6(a) and 6(b) suggest that, in Fig. 6(c), the narrow current peak is due to electron emission by the left-to-center and the center positive optical half cycles, and the broad peak is driven by the right-to-center positive optical half cycle. In Fig. 6(d), the single dominant current peak is mainly driven by the center positive optical half cycle of the laser field under strong dc bias.

\section{CONCLUSION}

In summary, we present a quantum analytical solution for few-cycle photoelectron emission from a dc-biased surface induced by Gaussian laser pulses, by solving the timedependent Schrödinger equation. Our calculations show the emitted charge per pulse oscillatorily increases as the laser pulse separation decreases due to varying coherence interaction of neighboring laser pulses. Our calculations recover the experimentally measured features of sinusoidal CEP modulation to photoelectron emission and vanishing CEP sensitivity with a $\pi$ phase shift in the optical-field regime under strong optical fields. Moreover, we find adding a large dc field greatly enhances the photoelectron current and shortens the current pulse. Future work will consider the effects of emitter tip geometry, surface roughness and defects, semiconductor or two-dimensional cathode material, nonequilibrium electron distribution due to laser heating, space charge effects, and the effects of dephasing of coherent polarizations in photoelectron emission.

\section{ACKNOWLEDGMENTS}

The work is supported by the Air Force Office of Scientific Research (AFOSR) YIP Grant No. FA9550-18-1-0061, and the Office of Naval Research (ONR) YIP Grant No. N0001420-1-2681.

\section{APPENDIX A: EXACT SOLUTION OF ELECTRON WAVE FUNCTION}

Following Truscott [24,53], the time-dependent potential for $x \geqslant 0$ may be written as $\Phi(x, t)=V(x)-x f(t)$, with $\quad V(x)=E_{F}+W_{\text {eff }}-e\left(F_{0}+F_{1} a_{0} \cos \phi\right) x, \quad$ and $f(t)=e \sum_{n=-\infty, n \neq 0}^{+\infty} F_{1} a_{n} \cos \left(\frac{n \pi}{L} t+\phi\right) \quad$ [cf. Eqs. (2) and (3) in the main text]. Thus, Eq. (4) in the main text can be transformed to the coordinate system $\xi$, $t$, where $\xi=x-q(t)$, the displacement $q(t)=\left(1 / m_{e}\right) \int^{t^{t}} p\left(t^{\prime}\right) d t^{\prime}$, and $p(t)=$ $\int^{t} f\left(t^{\prime}\right) d t^{\prime}$, by assuming that $\psi(x, t)=\phi(\xi, t) \chi(x, t)$, with $\chi(x, t)=\exp \left[-i E t / \hbar+i x p(t) / \hbar-\left(i / 2 \hbar m_{e}\right) \int^{t} p^{2}\left(t^{\prime}\right) d t^{\prime}\right]$, and $E$ is a constant. Then, putting the transformed potential energy $U(\xi, t)=E_{F}+W_{\text {eff }}-e\left(F_{0}+F_{1} a_{0} \cos \phi\right)[\xi+q(t)]$ and wave function $\psi(x, t)=\phi(\xi, t) \chi(x, t)$ into the time-dependent Schrödinger equation yields,

$$
i \hbar \frac{\partial \phi(\xi, t)}{\partial t}=\left[-\frac{\hbar^{2}}{2 m_{e}} \frac{\partial^{2}}{\partial \xi^{2}}+U(\xi, t)-E\right] \phi(\xi, t) .
$$


By separation of variables, Eq. (A1) can be solved to give

$$
\phi(\xi, t)=g(\xi) \exp \left[\sum_{n=-\infty, n \neq 0}^{+\infty} \frac{e^{2} a_{n} F_{1}\left(F_{0}+F_{1} a_{0} \cos \phi\right) \sin \left(n \omega_{E} t+\phi\right)}{i \hbar m_{e} n^{3} \omega_{E}^{3}}\right],
$$

where $g(\xi)=\operatorname{Ai}(-\eta)-i \operatorname{Bi}(-\eta) \quad$ is the solution of the equation $-\left(\hbar^{2} / 2 m_{e}\right) \partial^{2} g(\xi) / \partial \xi^{2}+$ $\left[E_{F}+W_{\text {eff }}-E-e\left(F_{0}+F_{1} a_{0} \cos \phi\right) \xi\right] g(\xi)=0, \quad$ with $\eta=\left[2 e m_{e}\left(F_{0}+F_{1} a_{0} \cos \phi\right) / \hbar^{2}\right]^{1 / 3}\left[\left(E-E_{F}-W_{\text {eff }}\right) / e\left(F_{0}+\right.\right.$ $\left.\left.F_{1} a_{0} \cos \phi\right)+\xi\right][49,50,54]$. From $\psi(x, t)=\phi(\xi, t) \chi(x, t)$, we obtain Eq. (5) in the main text, which is the exact solution to Eq. (4) upon using $E=\varepsilon+l \hbar \omega_{E}-U_{p}$, with $U_{p}=\sum_{n=-\infty, n \neq 0}^{+\infty} \frac{e^{2} F_{1}^{2}\left[a_{n}^{2}+a_{n} a_{n-n} \cos (2 \phi)\right]}{4 m_{e} n^{2} \omega_{E}^{2}}$.

For the special case of dc field $F_{0}=0$ and carrier-envelope phase $\phi=(2 n+1) \pi / 2$, with $n$ being an integer, the solution of $\psi(x, t)$ is revised by merely displacing $g(\xi)$ in Eq. (A2) with $\exp \left[i \xi \sqrt{2 m_{e} E} / \hbar\right]$.

\section{APPENDIX B: EVALUATION OF TRANSMISSION COEFFICIENT}

By imposing the boundary conditions that both the electron wave function $\psi(x, t)$ and its derivative $\partial \psi(x, t) / \partial x$ are continuous at $x=0$ and taking the Fourier transform, we obtain, in nondimensional quantities [24,27,28], $\bar{\varepsilon}=\varepsilon / W_{\text {eff }}, \bar{\omega}_{E}=\omega_{E} \hbar / W_{\text {eff }}, \bar{t}=t W_{\text {eff }} / \hbar, \bar{E}_{F}=E_{F} / W_{\text {eff }}, \bar{x}=$ $x / \lambda_{0}, \lambda_{0}=\sqrt{\hbar^{2} / 2 m_{e} W_{\text {eff }}}, \bar{F}_{0}=F_{0} e \lambda_{0} / W_{\text {eff }}, \bar{F}_{1}=F_{1} e \lambda_{0} / W_{\text {eff }}$, $\bar{U}_{p}=U_{p} / W_{\mathrm{eff}}$,

$$
2 \sqrt{\bar{\varepsilon}} \delta(k)=\sum_{l=-\infty}^{\infty} T_{l}\left[\sqrt{\bar{\varepsilon}+k \bar{\omega}_{E}} P_{l(l-k)}+Q_{l(l-k)}\right],
$$

where $\delta(k), P_{l(l-k)}$, and $Q_{l(l-k)}$ are

$$
\begin{aligned}
\delta(k) & = \begin{cases}1, & k=0 \\
0, & k \neq 0\end{cases} \\
P_{n l} & =\frac{1}{2 \pi} \int_{0}^{2 \pi} p_{n}\left(\bar{\omega}_{E} \bar{t}\right) e^{-i l \bar{\omega}_{E} \bar{t}} d\left(\bar{\omega}_{E} \bar{t}\right) \\
Q_{n l} & =\frac{1}{2 \pi} \int_{0}^{2 \pi} q_{n}\left(\bar{\omega}_{E} \bar{t}\right) e^{-i l \bar{\omega}_{E} \bar{t}} d\left(\bar{\omega}_{E} \bar{t}\right) \\
p_{n}\left(\bar{\omega}_{E} \bar{t}\right)= & \phi\left(\bar{\omega}_{E} \bar{t}\right)\left[\mathrm{Ai}\left(\alpha_{n}\right)-i \mathrm{Bi}\left(\alpha_{n}\right)\right] \\
q_{n}\left(\bar{\omega}_{E} \bar{t}\right)= & \phi\left(\bar{\omega}_{E} \bar{t}\right) z_{n}\left(\bar{\omega}_{E} \bar{t}\right), \\
\phi\left(\bar{\omega}_{E} \bar{t}\right)= & e^{i \frac{\bar{F}_{1}^{2}}{4} \bar{M}-2 i\left(\bar{F}_{0}+\bar{F}_{1} a_{0} \cos \phi\right) \bar{F}_{1} \bar{N}-i \frac{\bar{F}_{1}^{2}}{2 \bar{\omega}_{E}^{2}} \bar{R}} \\
z_{n}\left(\bar{\omega}_{E} \bar{t}\right)= & \bar{F}_{1}\left[\mathrm{Ai}^{2}\left(\alpha_{n}\right)-i \mathrm{Bi}\left(\alpha_{n}\right)\right] \bar{G}+\left(\bar{F}_{0}+\bar{F}_{1} a_{0} \cos \phi\right)^{1 / 3} \\
& \times\left[i \mathrm{Ai}^{\prime}\left(\alpha_{n}\right)+\mathrm{Bi}^{\prime}\left(\alpha_{n}\right)\right]
\end{aligned}
$$

where $\quad \bar{G}=\sum_{n=-\infty, n \neq 0}^{+\infty} \frac{a_{n} \sin \left(n \bar{\omega}_{E} \bar{t}+\phi\right)}{n \bar{\omega}_{E}}, \quad \bar{M}=\sum_{n=-\infty, n \neq 0}^{+\infty}$ $\frac{a_{n}^{2} \sin \left(2 n \bar{\omega}_{E} \bar{t}+2 \phi\right)+a_{n} a_{-n} \sin \left(2 n \bar{\omega}_{E} \bar{t}\right)}{n^{3} \bar{\omega}_{E}^{3}}, \quad \bar{N}=\sum_{n=-\infty, n \neq 0}^{+\infty} \frac{a_{n} \sin \left(n \bar{\omega}_{\bar{\omega}} \bar{t}+\phi\right)}{n^{3} \bar{\omega}_{E}^{3}}$, $\bar{R}=\sum_{n=-\infty, n \neq 0}^{+\infty} \quad \sum_{m=-\infty, m \neq 0, n,-n}^{+\infty} \frac{a_{m} a_{n}}{m n} \quad\left\{\frac{\sin \left[(n-m) \bar{\omega}_{E} \bar{t}\right]}{(n-m) \bar{\omega}_{E}}-\right.$ $\left.\frac{\sin \left[(n+m) \bar{\omega}_{E} \bar{t}+2 \phi\right]}{(n-m) \bar{\omega}_{E}}\right\}, \quad \alpha_{n}=-\left(\bar{F}_{0}+\bar{F}_{1} a_{0} \cos \phi\right)^{1 / 3}\left[\frac{\bar{E}_{n}}{\bar{F}_{0}+\bar{F}_{1} a_{0} \cos \phi}+\right.$ $\left.\sum_{l=-\infty, l \neq 0}^{+\infty} \frac{2 \bar{F}_{1} a_{l} \cos \left(l \bar{\omega}_{E} \bar{t}+\phi\right)}{l^{2} \bar{\omega}_{E}^{2}}\right]$, and $\bar{E}_{n}=\bar{\varepsilon}+n \bar{\omega}_{E}-\bar{E}_{F}-\bar{U}_{p}-$ 1. Here, $p_{n}\left(\bar{\omega}_{E} \bar{t}\right)$ and $q_{n}\left(\bar{\omega}_{E} \bar{t}\right)$ in Eqs. (B2c) and (B2d) denote the phase factor of the $n$ th-state wave function and of its spatial derivative at $\bar{x}=0$, respectively. $P_{n l}$ and $Q_{n l}$ are the $l$ th Fourier coefficient of $p_{n}\left(\bar{\omega}_{E} \bar{t}\right)$ and $q_{n}\left(\bar{\omega}_{E} \bar{t}\right)$, respectively. Thus, Eq. (B1) can be written as the following matrix operation,

$$
\left[\begin{array}{c}
0 \\
0 \\
\vdots \\
2 \sqrt{\bar{\varepsilon}} \\
\vdots \\
0 \\
0
\end{array}\right]=\left[\begin{array}{ccccc}
A_{(-N)[-N-(-N)]} & A_{(-N+1)[-N+1-(-N)]} & \cdots & A_{(N-1)[N-1-(-N)]} & A_{N[N-(-N)]} \\
A_{(-N)[-N-(-N+1)]} & A_{(-N+1)[-N+1-(-N+1)]} & \cdots & A_{(N-1)[N-1-(-N+1)]} & A_{N[N-(-N+1)]} \\
\vdots & \vdots & \ddots & \vdots & \vdots \\
A_{(-N)[-N-(N-1)]} & A_{(-N+1)[-N+1-(N-1)]} & \cdots & A_{(N-1)[N-1-(N-1)]} & A_{N[N-(N-1)]} \\
A_{(-N)(-N-N)} & A_{(-N+1)(-N+1-N)} & \cdots & A_{(N-1)(N-1-N)} & A_{(N)(N-N)}
\end{array}\right]\left[\begin{array}{c}
T_{-N} \\
T_{-N+1} \\
\vdots \\
T_{0} \\
\vdots \\
T_{N-1} \\
T_{N}
\end{array}\right],
$$

where $A_{(l)(l-k)}=\sqrt{\bar{\varepsilon}+k \bar{\omega}_{E}} P_{l(l-k)}+Q_{l(l-k)}$. If $N$ is large enough, we are able to get the converged value of the transmission coefficient $T_{n}$ (and therefore the reflection coefficient $R_{n}$ ) by solving the above matrix.

For the special case of dc field $F_{0}=0$ and carrier-envelope phase $\phi=(2 n+1) \pi / 2$, with $n$ being an integer, the transmission coefficient $T_{n}$ is still calculated from Eq. (B1) with $P_{n l}$ and $Q_{n l}$ unchanged, but with $p_{n}\left(\overline{\omega_{E}} \bar{t}\right)=$ $\phi\left(\bar{\omega}_{E} \bar{t}\right) \exp \left[i \sqrt{\bar{E}_{n}} \sum_{l=-\infty, l \neq 0}^{+\infty} \frac{2 \bar{F}_{1} a_{l} \cos \left(l \bar{\omega}_{E} \bar{t}+\phi\right)}{l^{2} \bar{\omega}_{E}^{2}}\right]$ and $q_{n}\left(\overline{\omega_{E}} \bar{t}\right)=p_{n}\left(\overline{\omega_{E}} \bar{t}\right)\left[\sqrt{\bar{E}_{n}}+\sum_{l=-\infty, l \neq 0}^{+\infty} \frac{\overline{\bar{F}_{1} a_{l} \sin \left(l \bar{\omega}_{E} \bar{t}+\phi\right)}}{l \bar{\omega}_{E}}\right]$.

[1] P. Hommelhoff, C. Kealhofer, and M. A. Kasevich, Ultrafast Electron Pulses from a Tungsten Tip Triggered by Low-
Power Femtosecond Laser Pulses, Phys. Rev. Lett. 97, 247402 (2006). 
[2] R. Ganter, R. Bakker, C. Gough, S. C. Leemann, M. Paraliev, M. Pedrozzi, F. Le Pimpec, V. Schlott, L. Rivkin, and A. Wrulich, Laser-Photofield Emission from Needle Cathodes for Low-Emittance Electron Beams, Phys. Rev. Lett. 100, 064801 (2008)

[3] M. Krüger, M. Schenk, and P. Hommelhoff, Attosecond control of electrons emitted from a nanoscale metal tip, Nature 475, 78 (2011).

[4] D. J. Park, B. Piglosiewicz, S. Schmidt, H. Kollmann, M. Mascheck, and C. Lienau, Strong Field Acceleration and Steering of Ultrafast Electron Pulses from a Sharp Metallic Nanotip, Phys. Rev. Lett. 109, 244803 (2012).

[5] L. Wimmer, G. Herink, D. R. Solli, S. V. Yalunin, K. E. Echternkamp, and C. Ropers, Terahertz control of nanotip photoemission, Nat. Phys. 10, 432 (2014).

[6] X. Xiong, Y. Zhou, Y. Luo, X. Li, M. Bosman, L. K. Ang, P. Zhang, and $\mathrm{L}$. Wu, Plasmon-enhanced resonant photoemission using atomically thick dielectric coatings, ACS Nano 14, 8806 (2020)

[7] D.-S. Yang, O. F. Mohammed, and A. H. Zewail, Scanning ultrafast electron microscopy, Proc. Natl. Acad. Sci. USA 107, 14993 (2010).

[8] S. Sun, X. Sun, D. Bartles, E. Wozniak, J. Williams, P. Zhang, and C.-Y. Ruan, Direct imaging of plasma waves using ultrafast electron microscopy, Struct. Dyn. 7, 064301 (2020).

[9] G. Sciaini and R. J. D. Miller, Femtosecond electron diffraction: Heralding the era of atomically resolved dynamics, Rep. Prog. Phys. 74, 096101 (2011).

[10] M. Müller, A. Paarmann, and R. Ernstorfer, Femtosecond electrons probing currents and atomic structure in nanomaterials, Nat. Commun. 5, 1 (2014).

[11] M. I. Stockman, M. F. Kling, U. Kleineberg, and F. Krausz, Attosecond nanoplasmonic-field microscope, Nat. Photonics 1, 539 (2007).

[12] M. T. Hassan, Attomicroscopy: From femtosecond to attosecond electron microscopy, J. Phys. B: At., Mol. Opt. Phys. 51, 032005 (2018).

[13] P. Dombi, Z. Pápa, J. Vogelsang, S. V. Yalunin, M. Sivis, G. Herink, S. Schäfer, P. Groß, C. Ropers, and C. Lienau, Strongfield nano-optics, Rev. Mod. Phys. 92, 025003 (2020).

[14] P. Zhang and Y. Y. Lau, Ultrafast and nanoscale diodes, J. Plasma Phys. 82, 595820505 (2016).

[15] P. Zhang, Á. Valfells, L. K. Ang, J. W. Luginsland, and Y. Y. Lau, 100 years of the physics of diodes, Appl. Phys. Rev. 4, 011304 (2017).

[16] J. Lin, P. Y. Wong, P. Yang, Y. Y. Lau, W. Tang, and P. Zhang, Electric field distribution and current emission in a miniaturized geometrical diode, J. Appl. Phys. 121, 244301 (2017)

[17] P. Hommelhoff, Y. Sortais, A. Aghajani-Talesh, and M. A. Kasevich, Field Emission Tip as a Nanometer Source of Free Electron Femtosecond Pulses, Phys. Rev. Lett. 96, 077401 (2006)

[18] C. Ropers, D. R. Solli, C. P. Schulz, C. Lienau, and T. Elsaesser, Localized Multiphoton Emission of Femtosecond Electron Pulses from Metal Nanotips, Phys. Rev. Lett. 98, 043907 (2007).

[19] R. Bormann, M. Gulde, A. Weismann, S. V. Yalunin, and C. Ropers, Tip-Enhanced Strong-Field Photoemission, Phys. Rev. Lett. 105, 147601 (2010).
[20] M. Schenk, M. Krüger, and P. Hommelhoff, Strong-Field Above-Threshold Photoemission from Sharp Metal Tips, Phys. Rev. Lett. 105, 257601 (2010).

[21] G. Herink, D. R. Solli, M. Gulde, and C. Ropers, Field-driven photoemission from nanostructures quenches the quiver motion, Nature 483, 190 (2012).

[22] M. Krüger, M. Schenk, P. Hommelhoff, G. Wachter, C. Lemell, and J. Burgdörfer, Interaction of ultrashort laser pulses with metal nanotips: A model system for strong-field phenomena, New J. Phys. 14, 085019 (2012).

[23] B. Piglosiewicz, S. Schmidt, D. J. Park, J. Vogelsang, P. Groß, C. Manzoni, P. Farinello, G. Cerullo, and C. Lienau, Carrierenvelope phase effects on the strong-field photoemission of electrons from metallic nanostructures, Nat. Photonics 8, 37 (2014).

[24] P. Zhang and Y. Y. Lau, Ultrafast strong-field photoelectron emission from biased metal surfaces: Exact solution to timedependent Schrödinger equation, Sci. Rep. 6, 19894 (2016).

[25] H. Yanagisawa, S. Schnepp, C. Hafner, M. Hengsberger, D. E. Kim, M. F. Kling, A. Landsman, L. Gallmann, and J. Osterwalder, Delayed electron emission in strong-field driven tunnelling from a metallic nanotip in the multi-electron regime, Sci. Rep. 6, 1 (2016).

[26] M. Förster, T. Paschen, M. Krüger, C. Lemell, G. Wachter, F. Libisch, T. Madlener, J. Burgdörfer, and P. Hommelhoff, Two-Color Coherent Control of Femtosecond Above-Threshold Photoemission from a Tungsten Nanotip, Phys. Rev. Lett. 117, 217601 (2016).

[27] Y. Luo and P. Zhang, Ultrafast strong-field photoelectron emission due to two-color laser fields, Phys. Rev. B 98, 165442 (2018).

[28] Y. Luo and P. Zhang, Analysis of Two-Color Laser-Induced Electron Emission from a Biased Metal Surface Using an Exact Quantum Mechanical Solution, Phys. Rev. Appl. 12, 044056 (2019).

[29] K. L. Jensen, Introduction to the Physics of Electron Emission, 1st ed. (Wiley, Hoboken, NJ, 2017).

[30] M. Pant and L. K. Ang, Time-dependent quantum tunneling and nonequilibrium heating model for the generalized Einstein photoelectric effect, Phys. Rev. B 88, 195434 (2013).

[31] D. J. Park and Y. H. Ahn, Ultrashort field emission in metallic nanostructures and low-dimensional carbon materials, Adv. Phys.: X 5, 1726207 (2020).

[32] F. Sirotti, N. Beaulieu, A. Bendounan, M. G. Silly, C. Chauvet, G. Malinowski, G. Fratesi, V. Véniard, and G. Onida, Multiphoton $k$-resolved photoemission from gold surface states with 800-nm femtosecond laser pulses, Phys. Rev. B 90, 035401 (2014).

[33] M. Reutzel, A. Li, B. Gumhalter, and H. Petek, Nonlinear Plasmonic Photoelectron Response of Ag(111), Phys. Rev. Lett. 123, 017404 (2019).

[34] M. Reutzel, A. Li, and H. Petek, Coherent Two-Dimensional Multiphoton Photoelectron Spectroscopy of Metal Surfaces, Phys. Rev. X 9, 011044 (2019).

[35] M. Reutzel, A. Li, and H. Petek, Above-threshold multiphoton photoemission from noble metal surfaces, Phys. Rev. B 101, 075409 (2020).

[36] M. Reutzel, A. Li, Z. Wang, and H. Petek, Coherent multidimensional photoelectron spectroscopy of ultrafast quasiparticle dressing by light, Nat. Commun. 11, 2230 (2020). 
[37] H. Yanagisawa, C. Hafner, P. Doná, M. Klöckner, D. Leuenberger, T. Greber, J. Osterwalder, and M. Hengsberger, Laser-induced field emission from a tungsten tip: Optical control of emission sites and the emission process, Phys. Rev. B 81, 115429 (2010).

[38] W. P. Putnam, R. G. Hobbs, P. D. Keathley, K. K. Berggren, and F. X. Kärtner, Optical-field-controlled photoemission from plasmonic nanoparticles, Nat. Phys. 13, 335 (2017).

[39] P. D. Keathley, W. P. Putnam, P. Vasireddy, R. G. Hobbs, Y. Yang, K. K. Berggren, and F. X. Kärtner, Vanishing carrier-envelope-phase-sensitive response in optical-field photoemission from plasmonic nanoantennas, Nat. Phys. 15, 1128 (2019).

[40] S. V. Yalunin, M. Gulde, and C. Ropers, Strong-field photoemission from surfaces: Theoretical approaches, Phys. Rev. B 84, 195426 (2011).

[41] H. R. Reiss, Effect of an intense electromagnetic field on a weakly bound system, Phys. Rev. A 22, 1786 (1980).

[42] F. H. M. Faisal, J. Z. Kamiński, and E. Saczuk, Photoemission and high-order harmonic generation from solid surfaces in intense laser fields, Phys. Rev. A 72, 023412 (2005).

[43] M. Pant and L. K. Ang, Ultrafast laser-induced electron emission from multiphoton to optical tunneling, Phys. Rev. B 86, 045423 (2012).

[44] S. V. Yalunin, G. Herink, D. R. Solli, M. Krüger, P. Hommelhoff, M. Diehn, A. Munk, and C. Ropers, Field localization and rescattering in tip-enhanced photoemission, Ann Phys. 525, L12 (2013).

[45] J. Li and U. Thumm, Semiclassical approach for solving the time-dependent Schrödinger equation in spatially inhomo- geneous electromagnetic pulses, Phys. Rev. A 101, 013411 (2020).

[46] Y. Zhou and P. Zhang, A quantum model for photoemission from metal surfaces and its comparison with the three-step model and fowler-DuBridge model, J. Appl. Phys. 127, 164903 (2020).

[47] S. Schilt and T. Südmeyer, Carrier-envelope offset stabilized ultrafast diode-pumped solid-state lasers, Appl. Sci. 5, 787 (2015).

[48] Y. Luo, J. Luginsland, and P. Zhang, Interference modulation of photoemission from biased metal cathodes driven by two lasers of the same frequency, AIP Adv. 10, 075301 (2020).

[49] R. H. Fowler and L. Nordheim, Electron emission in intense electric fields, Proc. R. Soc. London, Ser. A 119, 173 (1928).

[50] J. W. Gadzuk and E. W. Plummer, Field emission energy distribution (FEED), Rev. Mod. Phys. 45, 487 (1973).

[51] P. Zhang, Scaling for quantum tunneling current in nano- and subnano-scale plasmonic junctions, Sci. Rep. 5, 9826 (2015).

[52] U. De Giovannini, D. Varsano, M. A. L. Marques, H. Appel, E. K. U. Gross, and A. Rubio, Ab initio angleand energy-resolved photoelectron spectroscopy with timedependent density-functional theory, Phys. Rev. A 85, 062515 (2012).

[53] W. S. Truscott, Wave Functions in the Presence of a TimeDependent Field: Exact Solutions and Their Application to Tunneling, Phys. Rev. Lett. 70, 1900 (1993).

[54] Y. Zhou and P. Zhang, Theory of field emission from dielectric coated surfaces, Phys. Rev. Research 2, 043439 (2020). 